\title{
Efecto de tratamientos pregerminativos sobre la germinación de semillas de gulupa (Passiflora edulis Sims.), granadilla (Passiflora ligularis Juss.) y cholupa (Passiflora maliformis L.)
}

\section{Effect of pre-germination treatments on the germination of seeds of purple passion fruit (Passiflora edulis Sims.), sweet granadilla (Passiflora ligularis Juss.) and cholupa (Passiflora maliformis L.)}
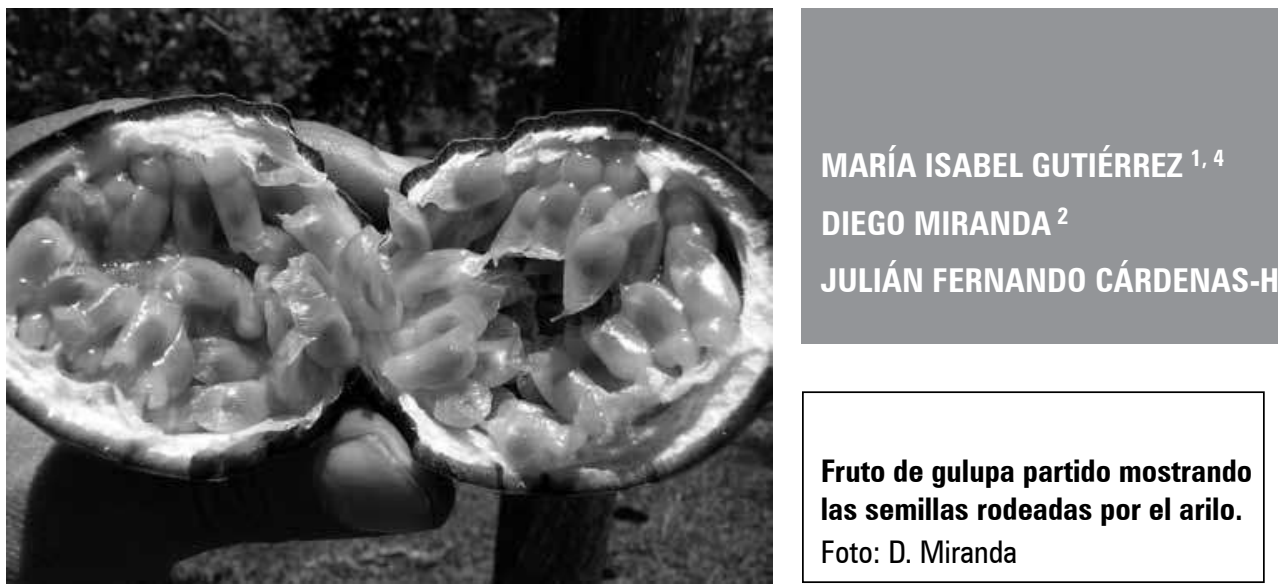

\section{RESUMEN}

El objetivo de este estudio fue determinar el efecto de la escarificación mecánica y química sobre la germinación de semillas de gulupa, granadilla y cholupa bajo condiciones de laboratorio (temperatura alterna $30 / 20^{\circ} \mathrm{C}[12 / 12$ horas], humedad relativa del $60 \%$ y en oscuridad durante 30 días). Semillas de las tres especies fueron sometidas a tres tipos de escarificación mecánica (despunte basal, despunte apical y punción de la testa), seis tratamientos de escarificación química (inmersión en $\mathrm{H}_{2} \mathrm{SO}_{4}$ al $49 \%$ y 98\%, durante 1, 3 ó 5 minutos) y comparadas con un testigo. Las semillas de gulupa sometidas a despunte basal y punción lograron aumentos significativos en la germinación ( $94 \%$ y $91 \%$ respectivamente) en comparación con los demás tratamientos y especies. Las semillas de granadilla también aumentaron su germinación al aplicar despunte basal y punción de la testa (52\% y 30\%). Ningún tratamiento favoreció la germinación de semillas de cholupa en comparación con el testigo.

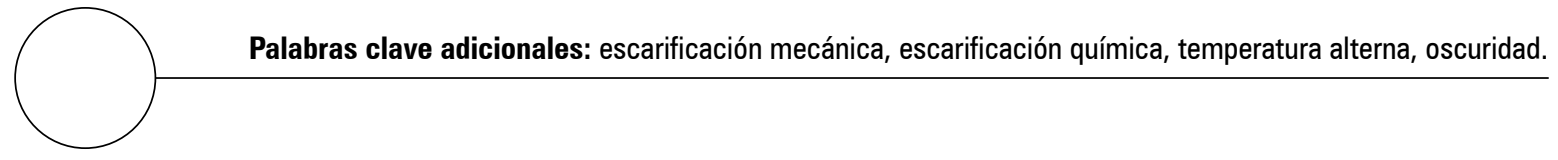

\footnotetext{
Facultad de Ciencias, Departamento de Biología, Universidad Nacional de Colombia, Bogotá (Colombia).

Facultad de Agronomía, Departamento de Agronomía, Universidad Nacional de Colombia, Bogotá (Colombia).

Facultad de Agronomía, Universidad Nacional de Colombia, Bogotá (Colombia).

4 Autor para correspondencia.migutierrezg@unal.edu.co
} 


\section{ABSTRACT}

The aim of this research was to determine the effect of chemical and mechanical scarification on germination of purple passionfruit, sweet granadilla and cholupa seeds under laboratory conditions (alternating temperature $30 / 20^{\circ} \mathrm{C}[12 / 12$ hours], relative humidity of $60 \%$ and in the dark for 30 days). The seeds of the three species seeds were subjected to three types of mechanical scarification (removal the basal point, removal the apical point and seed coat puncture), six treatments of chemical scarification (immersion in $\mathrm{H}_{2} \mathrm{SO}_{4}$ at $49 \%$ and $98 \%$, for 1,3 or 5 minutes) and compared to the control. Purple passionfruit seeds subjected to removal of the basal point and puncture achieved significant increases on germination ( $94 \%$ and $91 \%$ respectively) compared to other treatments and species. Sweet granadilla seeds also increased their germination after the removal of the basal point and seed coat puncture (52\% and 30\% respectively). No treatment favored the germination of cholupa seeds compared to the control.

Additional key words: mechanical scarification, chemical scarification, alternating temperature, dark.
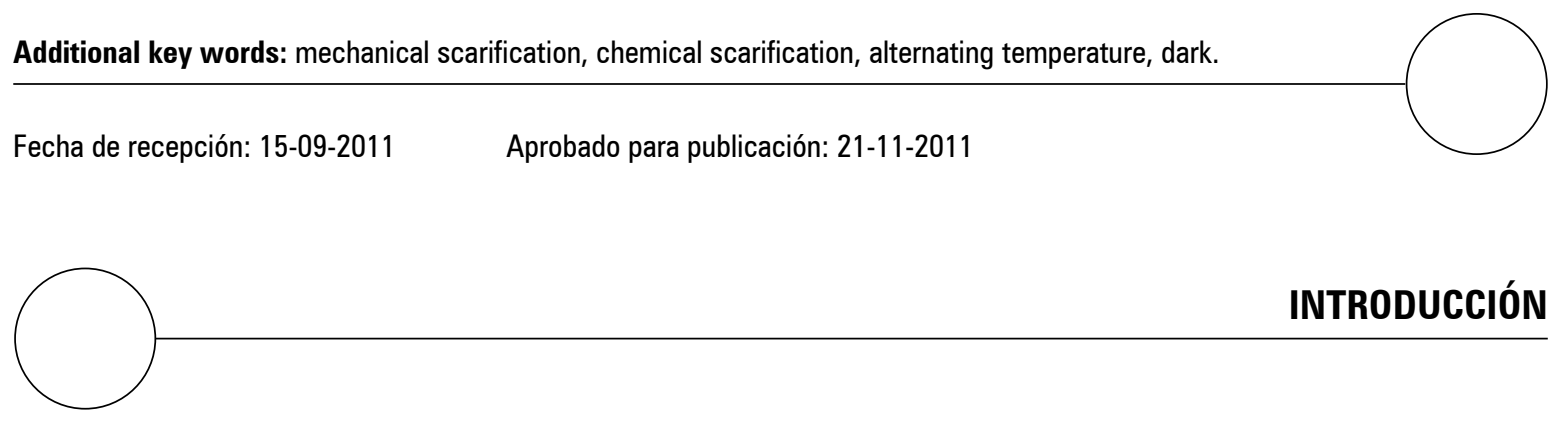

INTRODUCCIÓN

Colombia es el país con la mayor riqueza de $\mathrm{Pa}-$ sifloraceae, con 167 especies que se encuentran concentradas en la región andina, principalmente en los departamentos de Antioquia, Valle del Cauca, Cundinamarca, Quindío, Risaralda y Caldas (Ocampo et al., 2007). Los departamentos del Huila, Valle del Cauca, Cundinamarca, Antioquia y el Eje Cafetero han sido identificados como las principales regiones productoras del país (Cepass Huila, 2011).

Los frutos de granadilla, gulupa y cholupa tienen un alto potencial exportador, debido a sus características nutricionales (Rodríguez y Bermúdez, 2009), pero los cultivos de estas especies pueden presentar una baja productividad debido a que los métodos de propagación empleados actualmente generan materiales de siembra de baja calidad, con bajos porcentajes de germinación, baja población de plantas establecidas $\mathrm{y}$, por ende, bajos rendimientos.

Todas las pasifloras en general pueden ser propagadas sexual y asexualmente, pero se prefiere la utilización de las semillas como principal método de propagación, debido a que es el método más simple, más utilizado y menos costoso, que permite producir grandes cantidades de plantas, así como variabilidad entre plantas (Miranda et al., 2009). Ellis et al. (1985) han reportado que las semillas de pasifloras tienen latencia exógena, que es probablemente una combinación de latencia mecánica (cubierta seminal restringe el crecimiento radicular) y química (inhibidores en la cubierta seminal), por lo cual las semillas tienden a presentar una baja germinación. Según Pérez et al. (2005), la cubierta seminal de cholupa está entre 200 y $270 \mu \mathrm{m}$ de grosor y está constituida por cuatro capas de células.

Debido a la presencia de cubiertas seminales gruesas en la mayoría de semillas de pasifloráceas, es necesario aplicar tratamientos pregerminativos que permitan superar la latencia impuesta por las cubiertas y promuevan la germinación rápida y uniforme de las semillas. Esos tratamientos se conocen como escarificación y tienen como objetivo romper la cubierta de la se- 
milla para que el agua pueda entrar y pueda comenzar la germinación. Según Hartmann et al. (2002), tanto la escarificación mecánica como la escarificación química permiten romper la testa impermeable de las semillas.

Además del rompimiento de latencia, para que el proceso de germinación ocurra debe contarse con determinadas condiciones iniciales, como que la semilla sea viable, condiciones ambientales favorables (agua, temperatura, oxigeno y luz) y la ausencia de agentes patógenos (Barceló et al., 2001).

Entre los factores internos que afectan la germinación, se encuentra la viabilidad de las semillas que corresponde al periodo durante el cual las semillas conservan su capacidad para germinar, y el vigor que se define como el conjunto de propiedades que determinan el nivel de actividad y capacidad de las semillas durante la germinación y emergencia de las plántulas (ISTA, 1977). El primer factor se puede evaluar por medio de la prueba de viabilidad con sal de tetrazolio (2,3,5-trifenil cloruro de tetrazolio), que se basa en la actividad de las enzimas deshidrogenasas, las cuales catalizan las reacciones respiratorias en las mitocondrias durante la glucólisis y el ciclo de Krebs. Estas enzimas, particularmente las deshidrogenasas del ácido málico, reducen la sal de tetrazolio en los tejidos vivos a trifenil-formazan (compuesto rojo, estable y no difusible) (França et al., 1998). La actividad de las enzimas decrece paralelamente con la viabilidad de las semillas; por tanto, una coloración roja intensa es indicadora de la presencia de células vivas del embrión. En cambio, la no coloración o coloración rosa pálido del embrión son indicadoras que los tejidos no son viables (Moreno, 1984).

La temperatura es un factor decisivo en el proceso de la germinación, ya que influye sobre las enzimas que regulan la velocidad de las reacciones bioquímicas que ocurren en la semilla después de la imbibición. La actividad enzimática tiene lugar entre un máximo y un mínimo de temperatura, existiendo un óptimo intermedio. La temperatura óptima, puede definirse como la más adecuada para conseguir el mayor porcentaje de germinación en el menor tiempo posible (Bewley y Black, 1982).

Ellis et al. (1985) reportaron que las semillas dormantes de Passiflora spp. requieren escarificación y temperatura alterna $\left(20 / 30^{\circ} \mathrm{C}\right)$ para promover la germinación. La alternancia de temperatura puede producir cambios físicos en la cubierta de las semillas que favorecen la absorción de agua e inducen la germinación (Balaguera et al., 2010).

La luz es un factor importante que influye en la germinación de las semillas y en la emergencia de plántulas. Algunas semillas pueden ver inhibida su germinación una vez expuestas a la luz (fotoblásticas negativas) o promover su germinación (fotoblásticas positivas), aunque hay semillas cuya germinación es indiferente a este factor (Matilla, 2003).

Para promover la germinación en semillas de gulupa se han empleado diversos tratamientos: a) escarificación química con $\mathrm{H}_{2} \mathrm{SO}_{4}$ al 98\% durante 5 min o fermentación en solución de sacarosa al 10\%, generando porcentajes de germinación del 94 y 86,5\% respectivamente (Mabundza et al., 2010); b) imbibición en extracto acuoso de hojas secas de Casearia corymbosa durante $12 \mathrm{~h} \mathrm{a}$ una concentración de $2,5 \mathrm{mg} \mathrm{mL}^{-1}$ o imbibición en $\mathrm{GA}_{3}$ (giberrellic acid) durante $24 \mathrm{~h}$ a una concentración de $1 \mathrm{mg} \mathrm{mL}^{-1}$, alcanzando porcentajes de germinación del 81 y $94 \%$ respectivamente (Montero y Camargo, 2011).

En granadilla se han utilizado los siguientes tratamientos para facilitar la germinación: aplicación de $\mathrm{GA}_{3}(50,100,200$ o 400 mg L-1) y despunte basal bajo condiciones de oscuridad total a temperatura $\left(30 / 20^{\circ} \mathrm{C}\right)$, obteniendo porcentajes de germinación entre 42,75 y 76,5\%, y aplicación de $\mathrm{KNO}_{3}$ al 0,1\% para alcanzar un porcentaje de germinación del 96,33\% (Cárdenas, 2011). 
Para las semillas de cholupa, Ellis et al. (1985) recomiendan temperatura constante a $30^{\circ} \mathrm{C}$ o alternancia de temperatura: $20 / 30^{\circ} \mathrm{C}(12 / 12 \mathrm{~h})$.

La presente investigación evaluó el efecto de la escarificación mecánica y química, sobre la germinación de semillas de gulupa, granadilla y cholupa bajo condiciones controladas de laboratorio. Además se estudió el efecto de los tratamientos pregerminativos sobre la viabilidad de las semillas y se determinó el vigor del lote de semillas empleado en los ensayos de germinación.

\section{MATERIALES Y MÉTODOS}

Los frutos de gulupa y granadilla fueron colectados en el municipio de Íquira, vereda Los Andes, y los frutos de cholupa en el municipio de $\mathrm{Ri}$ vera, vereda Bajo Pedregal, en el departamento del Huila. Los frutos fueron colectados en estado 5 de maduración (Icontec, 1997, Pinzón et al., 2007) que se presenta después de la madurez fisiológica del fruto. Posteriormente los frutos fueron trasladados al laboratorio de Fisiología Vegetal de la Facultad de Agronomía, Universidad Nacional de Colombia, Bogotá, donde se extrajo la pulpa de los frutos y se colocó en recipientes de vidrio para su fermentación durante $48 \mathrm{~h}$. Una vez fermentada la pulpa, las semillas fueron extraídas manualmente, luego se colocaron sobre papel periódico y se dejaron secar a la sombra durante $48 \mathrm{~h}$, se almacenaron en frascos de vidrio y se mantuvieron a temperatura ambiente, para su posterior uso en los ensayos de germinación (Rivera et al., 2002).

Antes del montaje de los tratamientos, se realizó un test de tetrazolio para comprobar la viabilidad de las semillas. Se usaron 100 semillas en cuatro repeticiones, previamente embebidas en agua destilada a $30^{\circ} \mathrm{C}$ por $24 \mathrm{~h}$ (Gomes et al., 2011). Las semillas fueron cortadas longitudinalmente en uno de los extremos con bisturí, antes de su inmersión en una solución de cloruro 2, 3, 5-trifenil-2H-tetrazolio al 0,5\% (p/v) duran- te $24 \mathrm{~h}$, a $30^{\circ} \mathrm{C}$, bajo condiciones de oscuridad, según la metodología de Guevara et al. (s.f.). Las semillas fueron clasificadas como viables si el embrión presentaba una coloración roja intensa, y como no viables, si el embrión no presentaba esta coloración.

Para determinar el vigor de las semillas, se utilizó la prueba de conductividad eléctrica, donde se emplearon cuatro repeticiones por especie y cada repetición constó de 25 semillas. Estas fueron pesadas y sumergidas en $75 \mathrm{~mL}$ de agua destilada en vasos plásticos, y se mantuvieron durante 24 h en una cámara de germinación (Seedburo Achieva Germinator, Chicago, IL) a $25^{\circ} \mathrm{C}$. Después de este periodo, se determinó la conductividad eléctrica de la solución que contiene los lixiviados de las semillas, con un conductímetro ( $\mathrm{pH} /$ Cond 340i SET, WTW GmbH, Weilheim, Alemania) (Coelho, 2007). El valor de cada lectura de conductividad fue dividido por el respectivo peso de la muestra y los resultados fueron expresados en $\mu \mathrm{S} \mathrm{cm}^{-1} \mathrm{~g}^{-1}$ (AOSA, 1983).

Para evaluar el efecto de la escarificación mecánica y química sobre la germinación, semillas de gulupa, granadilla y cholupa previamente desinfectadas con Vitavax ${ }^{\circledR} 300$ (Proficol S.A., Bogotá) a una concentración de $2 \mathrm{~g} \mathrm{~L}^{-1}$ durante $3 \mathrm{~h}$, fueron sometidas a nueve tratamientos pregerminativos (despunte basal [DB] y despunte apical [DA] utilizando un cortaúñas, punción de la testa con alfiler [P], inmersión en $\mathrm{H}_{2} \mathrm{SO}_{4}$ al $49 \%$ y $98 \%$ durante 1, 3 y 5 min, respectivamente) y se incluyó un testigo por especie, con cinco repeticiones cada uno, usando 30 semillas por repetición. Cada repetición consistió en una caja de Petri con papel de filtro Munktell con 2 $\mathrm{mL}$ de agua destilada, en donde se colocaron las 30 semillas después de aplicar los respectivos tratamientos. Las cajas de Petri se colocaron en un fitotrón (Sanyo, modelo MLR-351H Anthony, TX) bajo temperatura alterna $30 / 20^{\circ} \mathrm{C}(12 / 12$ h), humedad relativa del $60 \%$ y en oscuridad durante $30 \mathrm{~d}$. Semanalmente, se cambió el papel de filtro y se llevó a cabo la evaluación de la ger- 
minación de las semillas tres veces por semana durante un mes, considerando germinada una semilla, si la radícula presentaba una longitud de $5 \mathrm{~mm}$ (Delanoy et al., 2006).

Con los datos obtenidos se calcularon las variables: porcentaje de germinación (PG), tiempo medio de germinación (TMG) y velocidad media de germinación (VMG) de acuerdo con Cárdenas (2011).

$$
P G=\left(N / N_{S}\right) \times 100
$$

donde: $N=$ número de semillas germinadas y $N_{S}=$ número de semillas totales.

$$
\begin{aligned}
T M G & =\sum_{i=1}^{k} n_{i} \times t_{i} / \sum_{i=1}^{k} n_{i} \\
V M G & =\sum\left(n_{i} / t_{i}\right)
\end{aligned}
$$

donde: $t_{\mathrm{i}}=$ tiempo en días, para la germinación en el i-ésimo día; $n_{\mathrm{i}}$ número de semillas germinadas en el i-ésimo día y $k$ : última germinación.

Una vez terminados los ensayos se realizó una clasificación de las semillas sin germinar (ISTA, 1996) y se procedió a realizar la prueba de viabilidad en estas semillas según la metodología de Guevara et al. (s.f.).

Para la prueba de conductividad eléctrica, se utilizó un diseño experimental de una sola vía, donde el factor correspondió a la especie y la variable dependiente fue la conductividad.

En las pruebas de tratamientos pregerminativos, se utilizó un diseño completamente al azar con arreglo factorial $3 \times 10$, donde el primer factor correspondió a la especie, y el segundo al tratamiento aplicado a la semilla.

Los datos que no presentaron normalidad según la prueba de Shapiro-Wilk, se transformaron con Raíz (x) (Molinero, 2003). Luego fueron sometidos a análisis de varianza y se realizó una prueba de comparación múltiple de medias por Tukey con un nivel de significancia del $5 \%$, utilizando el paquete estadístico Statistica v. 10 (StatSoft).

\section{RESULTADOS Y DISCUSIÓN}

\section{Viabilidad y vigor de las semillas}

La viabilidad de las semillas para cada una de las especies fue de $99 \%$, pues solo se identificó un embrión como no viable. Respecto a la conductividad eléctrica, se obtuvo el menor valor para las semillas de gulupa $\left(43,61 \mu \mathrm{S} \mathrm{cm}^{-1} \mathrm{~g}^{-1}\right)$, y para el caso de granadilla y cholupa las lecturas fueron de 107,49 y $102,20 \mu \mathrm{S} \mathrm{cm}^{-1} \mathrm{~g}^{-1}$, respectivamente. Al realizar un análisis de varianza para la variable conductividad de acuerdo con el factor especie, se concluye que la conductividad eléctrica determinada en los lixiviados de la semilla fue variable y dependiente de la especie.

La prueba de conductividad eléctrica se basa en la mayor o menor liberación de lixiviados por parte de las semillas (iones, azúcares, aminoácidos, entre otros) al medio de imbibición de acuerdo con la condición fisiológica de las mismas. Las semillas deterioradas o con bajo potencial fisiológico (menor vigor) son las que liberan mayor cantidad de lixiviados al medio (mayor conductividad), debido a la pérdida de la integridad de las membranas celulares (Craviotto y Arango, 2002; Vieira et al., 2002). De acuerdo con esta información, las semillas de granadilla y cholupa presentaron un menor vigor comparadas con gulupa, pues las semillas de gulupa presentaron una elevada germinación en comparación con las otras dos especies (tablas 1 a 3).

Según la prueba de tetrazolio, las semillas de gulupa, granadilla y cholupa, presentaron una alta capacidad germinativa, pero esto difiere de lo obtenido en los ensayos de germinación, con excepción de gulupa en algunos tratamientos de escarificación mecánica (DB y P). Las diferencias entre los porcentajes de viabilidad y germinación pueden deberse a la presencia de latencia en las 
semillas, infección de las semillas por hongos como se observó en algunos tratamientos de escarificación química y deterioro de las semillas durante la prueba de germinación (semillas muertas) (Moreno, 1984). Semillas de granadilla sometidas a DB bajo diferentes temperaturas (15, $20,25,25 / 15)$ en oscuridad total, presentaron un PG por debajo del porcentaje de semillas viables, lo cual puede estar relacionado con la existencia de latencia que pudo reprimir la germinación (Cárdenas, 2011). Las semillas de gulupa, granadilla y cholupa, en todos los tratamientos evaluados, presentaron un PG por debajo del porcentaje de viabilidad (tablas 1 a 3 ), lo cual puede indicar la presencia de latencia en semillas de esta especie, como fue reportado por Ellis et al. (1985), quienes sugieren que las semillas de pasifloras tienen latencia exógena.

\section{Ensayos de germinación}

El análisis de varianza para las variables PG, VMG y porcentaje de viabilidad de acuerdo con los factores: especie, tratamientos y sus interacciones, mostró que todos los factores afectaron significativamente las tres variables $(P \leq 0,05)$. De acuerdo con la prueba de Tukey, el PG pre- sentó diferencias significativas entre especies, la VMG no mostró diferencia significativa entre granadilla y cholupa, pero sí con gulupa, este mismo comportamiento se observa para la variable porcentaje de viabilidad (tablas 1 a 3 ).

\section{Porcentajes de germinación}

El DB y la punción fueron los mejores tratamientos para gulupa y granadilla, con PG del 94 y 91\% para gulupa, y PG del 52 y 30\% para granadilla (tablas 1 y 2). Respecto a cholupa, se alcanzó el mayor PG en el testigo (42\%), lo que indica, que ningún tratamiento pregerminativo evaluado favoreció la germinación en esta especie (tabla 3). Con los tratamientos de escarificación química se alcanzaron bajos porcentajes de germinación, para gulupa estos fluctuaron entre 0 y $27,5 \%$, en granadilla estuvieron entre 0 y $10 \%$, y en cholupa no hubo germinación (tablas 1 a 3).

Tanto en gulupa como en granadilla, el DB y la P mostraron los mayores PG, mientras que el DA y la escarificación química no favorecieron la germinación respecto al testigo (tablas 1 y 2). Que los porcentajes de germinación sean altos cuando se realiza despunte basal, se debe a que

Tabla 1. Germinación, VMG y viabilidad de semillas de gulupa, sometidas a oscuridad, temperatura alterna $30 / 20^{\circ} \mathrm{C}(12 / 12 \mathrm{~h})$ y $60 \%$ HR durante $30 \mathrm{~d}$, en nueve tratamientos comparados con el testigo.

\begin{tabular}{|l|c|c|c|}
\hline \multicolumn{1}{|c|}{ Tratamiento } & Germinación (\%) & VMG & 82 abcde \\
\hline Testigo & $73 \mathrm{abc}$ & $1,29 \mathrm{bc}$ & $95 \mathrm{ab}$ \\
\hline $\mathrm{DB}$ & $94 \mathrm{a}$ & $2,15 \mathrm{a}$ & $53 \mathrm{ghij}$ \\
\hline $\mathrm{DA}$ & $34 \mathrm{def}$ & $0,63 \mathrm{de}$ & $93 \mathrm{ab}$ \\
\hline $\mathrm{H}_{2} \mathrm{SO}_{4} 49 \% 1 \mathrm{~min}$ & $91 \mathrm{ab}$ & $1,6 \mathrm{ab}$ & $87 \mathrm{abc}$ \\
\hline $\mathrm{H}_{2} \mathrm{SO}_{4} 49 \% 3 \mathrm{~min}$ & $9 \mathrm{ghi}$ & $0,11 \mathrm{hijk}$ & $97 \mathrm{a}$ \\
\hline $\mathrm{H}_{2} \mathrm{SO}_{4} 49 \% 5 \mathrm{~min}$ & $27,5 \mathrm{fg}$ & $0,25 \mathrm{ghij}$ & $84 \mathrm{abcd}$ \\
\hline $\mathrm{H}_{2} \mathrm{SO}_{4} 98 \% 1 \mathrm{~min}$ & $0 \mathrm{k}$ & $0 \mathrm{~m}$ & $66 \mathrm{cdefg}$ \\
\hline $\mathrm{H}_{2} \mathrm{SO}_{4} 98 \% 3 \mathrm{~min}$ & $5 \mathrm{hijk}$ & $0,04 \mathrm{jklm}$ & $73 \mathrm{bcdef}$ \\
\hline $\mathrm{H}_{2} \mathrm{SO}_{4} 98 \% 5 \mathrm{~min}$ & $10 \mathrm{jjk}$ & $0,09 \mathrm{klm}$ & 64 defg \\
\hline
\end{tabular}

VMG, velocidad media de germinación (semillas germinadas/d); DB, despunte basal; DA, despunte apical; $P$, punción de la testa con alfiler. Letras distintas en cada columna indican diferencia significativa según la prueba de Tukey $(P \leq 0,05)$. 


\begin{tabular}{|c|c|c|c|}
\hline Tratamiento & Germinación (\%) & VMG & Viabilidad (\%) \\
\hline Testigo & 20 efg & 0,17 ghi & 84 abcd \\
\hline DB & $52 \mathrm{bcd}$ & $0,77 \mathrm{~cd}$ & $56 \mathrm{fgh}$ \\
\hline $\mathrm{DA}$ & 8 ghij & 0,1 hijkl & 60 efgh \\
\hline $\mathrm{P}$ & 30 def & 0,43 defg & 55 fghi \\
\hline $\mathrm{H}_{2} \mathrm{SO}_{4} 49 \% 1 \mathrm{~min}$ & $5 \mathrm{ijk}$ & $0,06 \mathrm{klm}$ & $95 a b$ \\
\hline $\mathrm{H}_{2} \mathrm{SO}_{4} 49 \% 3 \mathrm{~min}$ & 10 hijk & $0,13 \mathrm{ijk} / \mathrm{m}$ & $22 \mathrm{~m}$ \\
\hline $\mathrm{H}_{2} \mathrm{SO}_{4} 49 \% 5 \mathrm{~min}$ & $0 \mathrm{k}$ & $0 \mathrm{~m}$ & $63 \mathrm{efg}$ \\
\hline $\mathrm{H}_{2} \mathrm{SO}_{4} 98 \% 1 \mathrm{~min}$ & $5 \mathrm{jk}$ & $0,06 \mathrm{klm}$ & 66 cdefg \\
\hline $\mathrm{H}_{2} \mathrm{SO}_{4} 98 \% 3 \mathrm{~min}$ & $5 \mathrm{jk}$ & $0,06 \mathrm{klm}$ & 43 hijk \\
\hline $\mathrm{H}_{2} \mathrm{SO}_{4} 98 \% 5 \mathrm{~min}$ & $5 \mathrm{jk}$ & $0,06 \mathrm{klm}$ & $33 \mathrm{klm}$ \\
\hline
\end{tabular}

VMG, velocidad media de germinación (semillas germinadas/d); DB, despunte basal; DA, despunte apical; P, punción de la testa con alfiler. Letras distintas en cada columna indican diferencia significativa según la prueba de Tukey $(P \leq 0,05)$.

la emergencia de la radícula ocurre por la parte basal de la semilla o micrópilo (Schmidt, 2000), como se ha reportado en $P$. mollissima y $P$. ligularis, donde se obtuvieron altos valores de germinación al remover la parte basal de las semillas (Delanoy et al., 2006; Cárdenas, 2011). Aunque en $P$. tricuspis, se ha encontrado que el DB y el DA aumentan significativamente los porcentajes de germinación, pero el DA es significativamente mejor y la germinación es más rápida (Delanoy et al., 2006).

En cholupa ninguno de los tratamientos pregerminativos mejoró los porcentajes de germinación en comparación al testigo (tabla 3), por lo que se requiere explorar otros métodos que puedan favorecer la germinación en esta especie.

\section{Porcentajes de viabilidad}

Los mayores porcentajes de viabilidad (82-97\%) en gulupa se obtuvieron en el testigo, punción, DB e inmersión en $\mathrm{H}_{2} \mathrm{SO}_{4}$ al 49\% durante uno, tres y cinco minutos (tabla 1). En el caso de granadilla, en el testigo e inmersión en $\mathrm{H}_{2} \mathrm{SO}_{4}$ al $49 \%$ durante un minuto, se presentaron los mayores porcentajes de viabilidad (84-95\%) (tabla
2). Para cholupa, los mejores porcentajes de viabilidad (86-95,33\%) se consiguieron en el testigo e inmersión en $\mathrm{H}_{2} \mathrm{SO}_{4}$ al $49 \%$ durante 1,3 y 5 minutos (tabla 3).

En cholupa y granadilla se alcanzaron los menores porcentajes de viabilidad con los tratamientos de escarificación mecánica, debido a que los cotiledones y el embrión pudieron haber sufrido daños al realizar los cortes respectivos con el cortaúñas o por la incisión con el alfiler, que llevó a un alto porcentaje de semillas muertas, las cuales presentaron necrosis de los tejidos; y por algunas inmersiones en $\mathrm{H}_{2} \mathrm{SO}_{4}$ (tablas 2 y 3 ). Para el caso de gulupa, los más bajos porcentajes de viabilidad (53-73\%) fueron registrados en DA e inmersión en $\mathrm{H}_{2} \mathrm{SO}_{4}$ al $98 \%$ durante uno, tres y cinco minutos (tabla 1), posiblemente porque estos tratamientos causaron daños a los cotiledones y al embrión, como se explicó anteriormente.

En los casos que se presentaron porcentajes de germinación por debajo del estándar de viabilidad, puede deberse a la presencia de latencia (Ellis et al., 1985), pues la prueba de tetrazolio no diferencia entre semillas latentes y no latentes (Moreno, 1984). 


\begin{tabular}{|c|c|c|c|}
\hline Tratamiento & Germinación (\%) & VMG & Viabilidad (\%) \\
\hline Testigo & 42 cde & 0,6 def & 95,33 a \\
\hline DB & 19,33 efg & 0,29 efgh & $38 \mathrm{jkl}$ \\
\hline $\mathrm{DA}$ & $13,33 \mathrm{fgh}$ & $0,26 \mathrm{fgh}$ & $26 \mathrm{Im}$ \\
\hline $\mathrm{P}$ & 17,33 efg & 0,27 efgh & $26,67 \mathrm{Im}$ \\
\hline $\mathrm{H}_{2} \mathrm{SO}_{4} 49 \% 1 \mathrm{~min}$ & $0 \mathrm{k}$ & $0 \mathrm{~m}$ & $86 a b c$ \\
\hline $\mathrm{H}_{2} \mathrm{SO}_{4} 49 \% 3 \mathrm{~min}$ & $0 \mathrm{k}$ & $0 \mathrm{~m}$ & $87,33 \mathrm{ab}$ \\
\hline $\mathrm{H}_{2} \mathrm{SO}_{4} 49 \% 5 \mathrm{~min}$ & $0 \mathrm{k}$ & $0 \mathrm{~m}$ & $92,67 \mathrm{ab}$ \\
\hline $\mathrm{H}_{2} \mathrm{SO}_{4} 98 \% 1 \mathrm{~min}$ & $0 \mathrm{k}$ & $0 \mathrm{~m}$ & 52,67 ghij \\
\hline $\mathrm{H}_{2} \mathrm{SO}_{4} 98 \% 3 \mathrm{~min}$ & $0 \mathrm{k}$ & $0 \mathrm{~m}$ & 39,33 ijkl \\
\hline $\mathrm{H}_{2} \mathrm{SO}_{4} 98 \% 5 \mathrm{~min}$ & $0 \mathrm{k}$ & $0 \mathrm{~m}$ & $29,33 \mathrm{klm}$ \\
\hline
\end{tabular}

VMG, velocidad media de germinación (semillas germinadas/d); DB, despunte basal; DA, despunte apical; P, punción de la testa con alfiler. Letras distintas en cada columna indican diferencia significativa según la prueba de Tukey $(P \leq 0,05)$.

\section{Velocidad y tiempo medio de germinación}

En gulupa se alcanzó la mayor VMG con el tratamiento DB (2,15 semillas germinadas/día), seguido por punción con una VMG de 1,6 semillas por día (tabla 1). Al igual que en gulupa, en granadilla se alcanzaron las mayores VMG con DB y punción $(0,77$ y 0,43 semillas /día respectivamente) (tabla 2); y en cholupa con el testigo se obtuvo una VMG de 0,6 semillas germinadas/ día (tabla 3).

Los menores TMG (9,00 a 12,95 d) se observaron en los tratamientos $\mathrm{DB}, \mathrm{DA}$ y $\mathrm{P}$, así como en el testigo para gulupa, seguidos por $\mathrm{DB}, \mathrm{DA}$ y $\mathrm{P}$ para granadilla, con valores alrededor de $17 \mathrm{~d}, \mathrm{y}$ por último, en cholupa se observaron los mayores TMG entre 18 y $22 \mathrm{~d}$, aunque con el testigo de granadilla se alcanzó un TMG de 23,82 d (tabla 4).

Debido a que la mayoría de TMG no pudieron ser determinados para los tratamientos de escarificación química, no fue posible realizar un análisis de varianza con estos datos, pero se realizó un análisis de varianza para esta variable (TMG), de acuerdo con los factores: especie, tratamientos de escarificación mecánica y sus interacciones, donde se reveló que todos los

\begin{tabular}{|c|c|c|}
\hline Especie & Tratamiento & TMG \\
\hline Gulupa & Testigo & $12,95 \mathrm{~cd}$ \\
\hline Gulupa & DB & $9,21 \mathrm{~d}$ \\
\hline Gulupa & DA & $12 d$ \\
\hline Gulupa & $\mathrm{P}$ & $11,77 \mathrm{~d}$ \\
\hline Granadilla & Testigo & $23,82 \mathrm{a}$ \\
\hline Granadilla & DB & 17,4 bc \\
\hline Granadilla & $\mathrm{DA}$ & $17,4 \mathrm{bc}$ \\
\hline Granadilla & $P$ & $17,02 \mathrm{bc}$ \\
\hline Cholupa & Testigo & $22,34 a b$ \\
\hline Cholupa & DB & $21,08 a b$ \\
\hline Cholupa & $\mathrm{DA}$ & $18,32 a b$ \\
\hline Cholupa & $P$ & $20,82 a b$ \\
\hline
\end{tabular}

TMG, Tiempo medio de germinación (d); DB, despunte basal; DA, despunte apical; P, punción de la testa con alfiler.

Promedios con letras distintas indican diferencia significativa según la prueba de Tukey $(P \leq 0,05)$

factores afectaron significativamente la variable $(P \leq 0,05)$. La prueba de Tukey indica que la variable TMG no presentó diferencia significativa entre granadilla y cholupa, pero sí respecto a gulupa (tabla 4), que es el mismo comportamiento que se observó para las variables VMG y porcentaje de viabilidad. 
El despunte basal aumentó la VMG tanto en gulupa como en granadilla y generó una disminución en los TMG, respecto a los otros tratamientos de escarificación mecánica, aunque esto fue más significativo para gulupa. Según Cárdenas (2011), el DB aceleró los índices TMG y VMG en semillas de granadilla, pues el tratamiento permite superar la restricción mecánica impuesta por la cubierta promoviendo la apertura de la cubierta y, por tanto, el proceso de germinación se acelera.

\section{Clasificación semillas no germinadas}

Las semillas que no germinaron fueron catalogadas en tres grupos (vanas, muertas y frescas) se- gún la ISTA (1996); en el tratamiento DB en gulupa, $27,78 \%$ de las semillas fueron clasificadas como muertas y $72,22 \%$ como frescas; respecto a los demás tratamientos se obtuvieron porcentajes de semillas frescas por encima de 89\% (tabla $5)$. En el caso de granadilla, para $\mathrm{DB}$, se encontró $4,22 \%$ semillas vanas, $47,57 \%$ semillas muertas y 48,21\% semillas frescas, en los demás tratamientos el porcentaje de semillas frescas estuvo por encima de $81 \%$ (tabla 5 ). Los tratamientos DB, DA y $\mathrm{P}$ en cholupa presentaron porcentajes de semillas muertas entre 67,58 y $86,35 \%$, lo que se relaciona con la baja viabilidad presentada en estos tratamientos (tabla 3); respecto a los demás tratamientos se alcanzaron porcentajes de semillas frescas entre 92,91 y $100 \%$ (tabla 5).

\begin{tabular}{|c|c|c|c|c|}
\hline \multirow{2}{*}{ Especie } & \multirow{2}{*}{ Tratamiento } & \multicolumn{3}{|c|}{ Semillas (\%) } \\
\hline & & Vanas & Muertas & Frescas \\
\hline Gulupa & Testigo & 0 & 10,67 & 89,33 \\
\hline Gulupa & $\mathrm{DB}$ & 0 & 27,78 & 72,22 \\
\hline Gulupa & $\mathrm{DA}$ & 0 & 9,51 & 90,49 \\
\hline Gulupa & $\mathrm{P}$ & 0 & 0 & 100 \\
\hline Gulupa & $\mathrm{H}_{2} \mathrm{SO}_{4} 49 \% 1 \mathrm{~min}$ & 0 & 0 & 100 \\
\hline Gulupa & $\mathrm{H}_{2} \mathrm{SO}_{4} 49 \% 3 \mathrm{~min}$ & 0 & 0 & 100 \\
\hline Gulupa & $\mathrm{H}_{2} \mathrm{SO}_{4}^{4} 49 \% 5 \mathrm{~min}$ & 0 & 0 & 100 \\
\hline Gulupa & $\mathrm{H}_{2} \mathrm{SO}_{4} 98 \% 1 \mathrm{~min}$ & 0 & 0 & 100 \\
\hline Gulupa & $\mathrm{H}_{2} \mathrm{SO}_{4} 98 \% 3 \mathrm{~min}$ & 0 & 1 & 99 \\
\hline Gulupa & $\mathrm{H}_{2} \mathrm{SO}_{4} 98 \% 5 \mathrm{~min}$ & 0 & 0 & 100 \\
\hline Granadilla & Testigo & 2,58 & 2,5 & 94,92 \\
\hline Granadilla & $\mathrm{DB}$ & 4,22 & 47,57 & 48,21 \\
\hline Granadilla & $\mathrm{DA}$ & 0 & 18,48 & 81,52 \\
\hline Granadilla & $\mathrm{P}$ & 0 & 10,55 & 89,45 \\
\hline Granadilla & $\mathrm{H}_{2} \mathrm{SO}_{4} 49 \% 1 \mathrm{~min}$ & 0 & 0 & 100 \\
\hline Granadilla & $\mathrm{H}_{2} \mathrm{SO}_{4} 49 \% 3 \mathrm{~min}$ & 0 & 0 & 100 \\
\hline Granadilla & $\mathrm{H}_{2} \mathrm{SO}_{4} 49 \% 5 \mathrm{~min}$ & 0 & 0 & 100 \\
\hline Granadilla & $\mathrm{H}_{2} \mathrm{SO}_{4}^{4} 98 \% 1 \mathrm{~min}$ & 0 & 0 & 100 \\
\hline Granadilla & $\mathrm{H}_{2} \mathrm{SO}_{4} 98 \% 3 \mathrm{~min}$ & 0 & 0 & 100 \\
\hline Granadilla & $\mathrm{H}_{2} \mathrm{SO}_{4} 98 \% 5 \mathrm{~min}$ & 0 & 0 & 100 \\
\hline Cholupa & Testigo & 1 & 6,09 & 92,91 \\
\hline Cholupa & $\mathrm{DB}$ & 0 & 67,58 & 32,42 \\
\hline Cholupa & $\mathrm{DA}$ & 0 & 80,11 & 19,89 \\
\hline Cholupa & $\mathrm{P}$ & 0 & 86,35 & 13,65 \\
\hline Cholupa & $\mathrm{H}_{2} \mathrm{SO}_{4} 49 \% 1 \mathrm{~min}$ & 1,33 & 0 & 98,67 \\
\hline Cholupa & $\mathrm{H}_{2} \mathrm{SO}_{4}^{4} 49 \% 3 \mathrm{~min}$ & 0 & 0 & 100 \\
\hline Cholupa & $\mathrm{H}_{2} \mathrm{SO}_{4} 49 \% 5 \mathrm{~min}$ & 0,67 & 0 & 99,33 \\
\hline Cholupa & $\mathrm{H}_{2} \mathrm{SO}_{4} 98 \% 1 \mathrm{~min}$ & 0,67 & 0 & 99,33 \\
\hline Cholupa & $\mathrm{H}_{2} \mathrm{SO}_{4} 98 \% 3 \mathrm{~min}$ & 0 & 0 & 100 \\
\hline Cholupa & $\mathrm{H}_{2} \mathrm{SO}_{4} 98 \% 5 \mathrm{~min}$ & 0 & 0 & 100 \\
\hline
\end{tabular}

DB, despunte basal; DA, despunte apical; P, punción de la testa con alfiler. 
Acorde con la clasificación de semillas no germinadas en las tres especies (tabla 5), se puede observar un aumento en la cantidad de semillas muertas con las escarificaciones mecánicas, mientras que con las escarificaciones químicas no, lo cual sugiere que las prácticas químicas son menos dañinas, posiblemente porque la cubierta de la semilla no permitió que el ácido penetrara y dañara el embrión, a las concentraciones de $\mathrm{H}_{2} \mathrm{SO}_{4}$ y tiempos de inmersión evaluados.

\section{CONCLUSIONES}

Bajo condiciones de oscuridad y temperatura alterna $\left(30 / 20^{\circ} \mathrm{C}\right)$ en laboratorio se pueden obtener aumentos en los porcentajes de germinación de semillas de gulupa y granadilla al aplicar despunte basal o punción de la testa, en comparación al testigo. Se recomienda realizar investigaciones con semillas de cholupa, donde se exploren otros tratamientos pregerminativos que puedan incidir de manera significativa en la germinación de las semillas.

\section{AGRADECIMIENTOS}

A la Dirección de Investigación Sede Bogotá (DIB) por el apoyo financiero, al igual que a Colciencias por la financiación otorgada al proyecto de investigación "Evaluación de tecnologías innovadoras para la obtención de material vegetal de siembra en cultivos de maracuyá (Passiflora edulis Sims.) y granadilla (Passiflora ligularis Juss.) para mejorar su productividad y competitividad en el departamento del Huila".

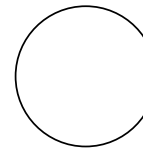

AOSA. 1983. Association of Official Seed Analysts. Seed vigour testing handbook. Contribution $\mathrm{N}^{\circ}$. 32. Lincoln, NE.

Balaguera, H., J. Álvarez y J. Cárdenas. 2010. Efecto de la estratificación fría y la cobertura plástica en semillas de gulupa (Passiflora edulis Sims.) para la obtención de plántulas. Rev. UDCA Act. \& Div. Cient. 13(2), 89-97.

Barceló, J., G. Nicolás, B. Sabater y R. Sánchez. 2001. Fisiología vegetal. Ediciones Pirámide, Madrid.

Bewley, J. y M. Black. 1982. Physiology and biochemistry of seeds in relations to germination. Vol. 2. Viability, dormancy and environmental control. Springer-Verlag, Berlin.

Cárdenas, J. 2011. Morfología y tratamientos pregerminativos de semillas de granadilla (Passiflora ligularis Juss.). Tesis de maestría. Facultad de Agronomía, Universidad Nacional de Colombia, Bogotá.

Cepass Huila. 2011. Conformación del Consejo Nacional de Pasifloras. En: Boletín No. 8 Vigilancia Tecnoló-

\section{REFERENCIAS BIBLIOGRÁFICAS}

gica, http://www.cepasshuila.org.co/cms/images/ pdf/b8/index.html; consulta: noviembre de 2011.

Coelho, A., S. Ferreira, V. Pio y S.M. Vinícius. 2007. Estádio de maturação e qualidade de sementes após repouso de frutos de maracujá amarelo. Rev. Bras. Sementes 29(3), 67-76.

Craviotto, R. y M. Arango. 2002. Sin semilla de calidad ningún sistema es sustentable. En: Instituto $\mathrm{Na}$ cional de Tecnología Agropecuaria, http://www. inta.gov.ar/oliveros/info/documentos/dia_campo/ artic11.htm; consulta: noviembre de 2011.

Delanoy, M., P. Van Damme, X. Scheldeman y J. Beltran. 2006. Germination of Passiflora mollissima (Kunth) L.H.Bailey, Passiflora tricuspis Mast. and Passiflora nov sp. seeds. Sci. Hortic. 110, 198-203.

Ellis, R., T. Hong y E. Roberts. 1985. Handbook of seed technology for genebanks. Vol. II: Compendium of specific germination. Information and test recommendations. International Board for Plant Genetic Resources (IBPGR), Roma. 
França, N., F. Krzyzanowski y N. Costa. 1998. El test de tetrazolio en semillas de soja. Londrina, Brasil: Embrapa-CNPSo. Documento 117.

Gomes, J., L. Castelo, R. Caldas, A. Nassif y S. Carvalho. 2011. Germinação de sementes de Passiflora Setacea e dormência induzida pelo armazenamento. Rev. Bras. Sementes 33(1), 80-85.

Guevara, C., J. Ospina, L. Caicedo, V. Barney y G. Coppens D'eeckenbrugge. s.f. Seed cryopreservation in three Passiflora species. En: CIAT, ftp://ciat.cgiar. org/ipgri/Templates\%20Bioversity/crioPasiflora. doc; consulta: septiembre de 2011.

Hartmann, H., D. Kester, F. Davies y R. Geneve. 2002. Plant propagation: principles and practices. $7^{\text {th }} \mathrm{ed}$. Prentice Hall, New Jersey, NJ.

Icontec. 1997. Norma técnica Colombiana (NTC) 4101. Frutas frescas granadilla, especificaciones. Instituto Técnico Colombiano de Normas Técnicas y Certificación, Bogotá.

ISTA. 1977. International Seed Testing Association. Rules for testing seeds. Bassersdorf, Suiza. ISTA. 1996. International Seed Testing Association. International rules for seed testing. Seed Sci. Technol. 24 (Supplement). pp. 1-343.

Mabundza, R., P. Wahome y M. Masarirambi. 2010. Effects of different pre-germination treatment methods on the germination of passion (Passiflora edulis) seeds. J. Agric. Soc. Sci. 6, 57-60.

Matilla, A. 2003. Capítulo 29: Ecofisiología de la germinación de semillas. pp. 901-922. En: Reigosa, M.J., N. Pedrol y A. Sánchez (eds.). La ecofisiología vegetal: una ciencia de síntesis. Ediciones Paraninfo, Madrid.

Miranda, D., M. Perea y S. Magnitskiy. 2009. Propagación de especies pasifloráceas. pp. 69-96. En: Miranda D.; G. Fischer; C. Carranza; S. Magnitskiy; F. Casierra-Posada; W. Piedrahíta y L. Flórez (eds.). Cultivo, poscosecha y comercialización de las pasifloráceas en Colombia: maracuyá, granadilla, gulupa y curuba. Sociedad Colombiana de Ciencias Hortícolas, Bogotá.
Molinero, L. 2003. ¿Y si los datos no siguen una distribución normal?. En: SEH-LELHA, http://www. seh-lelha.org/noparame.htm; consulta: noviembre de 2011.

Montero, D. y J. Camargo. 2011. Efecto del extracto de Caseira corymbosa sobre la germinación de Passiflora edulis var. edulis. Rev. Bras. Agroecol. 6(2), 63-69.

Moreno, E. 1984. Análisis físico y biológico de semillas agrícolas. 3a. ed. Universidad Nacional Autónoma de México, Mécico.

Ocampo, P., G. Coppens d'Eeckenbrugge,, M. Restrepo, A. Jarvis, M. Salazar y C. Caetano. 2007. Diversity of Colombian Passifloraceae: biogeography and an updated list for conservation. Biota Colomb. 8(1), $1-45$.

Pérez, C., M. Escala y S. Tillett. 2005. Anatomía de la cubierta seminal en ocho especies de Passiflora L., subgénero Passiflora. Acta Bot. Venez. 28(2), 337-348.

Pinzón, I.M.P., G. Fischer y G. Corredor. 2007. Determinación de los estados de madurez del fruto de la gulupa (Passiflora edulis Sims.). Agron. Colomb. 25(1), 83-95.

Rivera, B., D. Miranda, L. Ávila, A. Nieto. 2002. Manejo integral del cultivo de la granadilla (Passiflora ligularis Juss.). Editorial Litoas, Manizales, Colombia.

Rodríguez, L. y L. Bermúdez. 2009. Economía y gestión del sistema de producción de pasifloráceas en Colombia. pp. 303-326. En: Miranda D.; G. Fischer; C. Carranza; S. Magnitskiy; F. Casierra-Posada; W. Piedrahíta y L. Flórez (eds.). Cultivo, poscosecha y comercialización de las pasifloráceas en Colombia: maracuyá, granadilla, gulupa y curuba. Sociedad Colombiana de Ciencias Hortícolas, Bogotá.

Schmidt, L. 2000. Guide to handling of tropical and subtropical forest seed. Danida Forest Seed Centre, Humlebaek, Dinamarca.

Vieira, R., A. Penariol, D. Perecin y M. Panabianco. 2002. Condutividade elétrica e teor de água inicial das sementes de soja. Pesq. Agropec. Bras. 37(9), 1333 1338. 